\title{
A rigid Boolean algebra that admits the elimination of $Q_{1}^{2}$
}

\author{
by
}

\author{
Heike Mildenberger (Bonn)
}

\begin{abstract}
Using $\diamond$, we construct a rigid atomless Boolean algebra that has no uncountable antichain and that admits the elimination of the Malitz quantifier $Q_{1}^{2}$.
\end{abstract}

1. Introduction. Malitz quantifiers are introduced in [Mag-Mal]. Let us recall the semantics of $Q_{\alpha}^{n}, n \geq 1, \alpha \in$ ORD: $\mathfrak{A} \vDash Q_{\alpha}^{n} x \phi(\bar{a}, \stackrel{n}{x})$ iff there is a subset $H$ of $A$ such that $\operatorname{card}(H) \geq \aleph_{\alpha}$ and $\mathfrak{A} \vDash \phi(\bar{a}, h)$ for all pairwise different $h_{0}, h_{1}, \ldots, h_{n-1} \in H$. Such a set $H$ is called a homogeneous set for $\phi(\bar{a}, \stackrel{n}{x})$. Baldwin and Kueker [Bal-Ku], Rothmaler and Tuschik [Ro-Tu], Bürger [Bü] and Koepke [Ko] consider the question of elimination of some of these quantifiers in certain theories or structures. [Ro-Tu] shows that any saturated model allows the elimination of all $Q_{\alpha}^{n}, \alpha \in \mathrm{ORD}, n \geq 1$.

Saturated models with two elements of the same type are not rigid. On the other hand, there are $\mathcal{L}_{\omega \omega}\left(Q_{1}^{2}\right)$-sentences $\phi$ that have only rigid models and that are satisfiable under $\mathrm{CH}$ (see $[\mathrm{Ot}]$, [Mil]). We consider

$$
\begin{aligned}
\phi:= & \text { "the structure is a Boolean algebra with } 0 \neq 1 " \\
& \wedge \forall x\left(x \neq 0 \rightarrow Q_{1} y y \subseteq x\right) \wedge \neg Q_{1}^{2} x y x \not \subseteq y .
\end{aligned}
$$

[Ba-Ko, Theorem 5(a)] shows that all models of $\phi$ are rigid. The search for a model of $\phi$ that contains two different elements of the same $\mathcal{L}_{\omega \omega}\left(Q_{1}^{2}\right)$-type leads, under $\diamond$, to a model of $\phi$ that admits the elimination of $Q_{1}^{2}$ and in which therefore any two elements $\neq 0,1$ have the same $\mathcal{L}_{\omega \omega}\left(Q_{1}^{2}\right)$-type.

$\mathrm{In} \mathrm{ZFC}+\diamond$ and even in $\mathrm{ZFC}+\mathrm{CH}$ there are various constructions of uncountable Boolean algebras with no uncountable antichains and with some other algebraic properties (see [Ba-Ko], [Sh], [Ru], but also [Ba]). In the course of showing that additional tasks may be fulfilled along the way given in [Ba-Ko], we get a partition of all formulas $\phi(\stackrel{r}{z}, x, y) \in \mathcal{L}_{\omega \omega}\left(Q_{1}^{2}\right), r \in \omega$, into two classes $\Phi_{1}$ and $\Phi_{2}$ such that 
1. The methods of $[\mathrm{Ba}-\mathrm{Ko}]$ are applicable to any $\phi(\underset{z}{r}, x, y) \in \Phi_{1}$. They will allow us to show that the homogeneous sets for any $\phi(\stackrel{r}{z}, x, y) \in \Phi_{1}$ will grow only during countably many steps in the chain which we build in the next section.

2. For any Boolean algebra $\mathfrak{A}$ with $\mathfrak{A} \vDash \forall x \neq 0 Q_{1} y y \subseteq x$ and any $\phi(\stackrel{r}{z}, x, y) \in \Phi_{2}: \mathfrak{A} \vDash \exists z Q_{1}^{2} x y \phi(\stackrel{r}{z}, x, y)$.

" $\phi(\stackrel{r}{z}, x, y) \in \Phi_{1}$ " will be shown to be equivalent under the first order theory of atomless Boolean algebras to a first order formula with its free variables among $z_{0}, z_{1}, \ldots, z_{r-1}$. The consideration of the possible quantifierfree types of the $\underset{z}{z}$ leads to a procedure for eliminating $Q_{1}^{2}$.

\section{The construction}

Notation. We will use $\mathfrak{A}, \mathfrak{B}, \mathfrak{B}_{\alpha}$ to denote Boolean algebras. Boolean

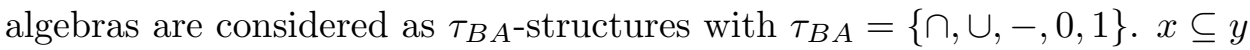
is written for $x \cap y=x, \subset$ means strict inclusion, $x \backslash y$ is used for $x \cap(-y)$. $\mathcal{P}(\omega)$ denotes the powerset algebra of $\omega$. For $\mathfrak{A} \subseteq \mathcal{P}(\omega)$ we often write $A$ for $\mathfrak{A}$. The interpretations of the $\tau_{B A}$-symbols in $\mathcal{P}(\omega)$ are denoted by the symbols themselves.

$a, b \in A$ are comparable (in $\mathfrak{A}$ ) iff $a \subseteq^{\mathfrak{A}} b$ or $b \subseteq^{\mathfrak{A}} a . \quad C \subseteq \mathfrak{A}$ is a chain (an antichain) iff any two distinct elements of $C$ are comparable (not comparable). For $a \subset^{\mathfrak{A}} b \in A$ let $(a, b)_{A}:=\left\{c \in A \mid a \subset^{\mathfrak{A}} c \subset^{\mathfrak{A}} b\right\}$.

Using $\diamond$, we shall construct a Boolean algebra $\mathfrak{B}$ such that $\mathfrak{B}$ is a model of the sentence $\phi$ from the introduction and $\mathfrak{B}$ admits the elimination of $Q_{1}^{2}$. As the construction of our Boolean algebra $\mathfrak{B}$ follows the pattern of [Ba-Ko], we restrict ourselves to a short description, heavily referring to [Ba-Ko].

Inductively on $\alpha \in \omega_{1}$, we shall build a chain $\left(\mathfrak{B}_{\alpha}, M_{\alpha}\right)_{\alpha \in \omega_{1}}$, where the $\mathfrak{B}_{\alpha}$ are countable atomless subalgebras of $\mathcal{P}(\omega)$ and each $M_{\alpha+1}$ is a countable collection of pairs $(M, \phi(\bar{c}, x, y))$, where $M \subseteq B_{\alpha}$ and $\phi(\bar{c}, x, y)$ is a quantifierfree (qf) $\mathcal{L}_{\omega \omega}\left[\tau_{B A}\right]$-formula with a property that will be defined later on, and $\bar{c}$ are elements of $B_{\alpha}$. At limit steps we take unions. $\mathfrak{B}_{\alpha+1}$ will be the Boolean algebra that is generated by $B_{\alpha} \cup\left\{x_{\alpha}\right\}$ in $\mathcal{P}(\omega)$, where the $x_{\alpha}$ is chosen by the same forcing $P\left(B_{\alpha}\right)$ as in [Ba-Ko], namely: $P\left(B_{\alpha}\right)=$ $\left\{(a, b)_{B_{\alpha}} \mid a \subset b \in B_{\alpha}\right\},\left(a^{\prime}, b^{\prime}\right)_{B_{\alpha}} \leq^{P\left(B_{\alpha}\right)}(a, b)_{B_{\alpha}}$ iff $a \subseteq a^{\prime} \subset b^{\prime} \subseteq b$.

We shall define $D_{A}(M, \phi(\bar{c}, x, y), e, f)$ and $M_{\alpha+1}$. Then we take a $\left\{D_{A}(M, \phi(\bar{c}, x, y), e, f) \mid e, f \in B_{\alpha},(M, \phi(\bar{c}, x, y)) \in M_{\alpha+1}\right\}$-generic subset $\left\{\left(a_{n}, b_{n}\right) \mid n \in \omega\right\}$ of $P\left(B_{\alpha}\right)$ such that $\left\{\left(a_{n}, b_{n}\right) \mid n \in \omega\right\}$ additionally satisfies the properties described in [Ba-Ko] and set $x_{\alpha}=\bigcup\left\{a_{n} \mid n \in \omega\right\}$. In [Ba-Ko], $M_{\alpha+1}$ is chosen so that chains and antichains are countable. Our $M_{\alpha+1}$ differs from that of $[\mathrm{Ba}-\mathrm{Ko}]$, because we also want all homogeneous sets for 
any $\phi(\stackrel{r}{z}, x, y) \in \Phi_{1}$ to be countable. The next items are the generalizations of the corresponding points of [Ba-Ko].

Definition 2.1. Let $A \subseteq \mathcal{P}(\omega)$ and $\bar{c}, e, f \in A$. Let $\phi(\bar{z}, x, y)$ be qf.

(i) $D_{A}(M, \phi(\bar{c}, x, y), e, f):=\left\{(a, b)_{A} \in P(A) \mid\right.$ for any $u \in(a, b)_{\mathcal{P}(\omega)}$ one of the following points is true:

1. $(u \cap e) \cup(f \backslash u) \in M$.

2. There is some $y \in M$ such that

$$
\mathcal{P}(\omega) \vDash \neg \phi(\bar{c},(u \cap e) \cup(f \backslash u), y) \vee \neg \phi(\bar{c}, y,(u \cap e) \cup(f \backslash u))\} .
$$

(ii) $M$ is called maximally homogeneous for $\phi(\bar{c}, x, y)$ in $\mathfrak{A}$ iff $M \subseteq A$ is homogeneous for $\phi(\bar{c}, x, y)$ and for all $a \in A \backslash M$ there is some $b \in M$ such that $\mathfrak{A} \vDash \neg \phi(\bar{c}, a, b) \vee \neg \phi(\bar{c}, b, a)$.

(iii) $\phi(\bar{c}, x, y)$ is small in $\mathfrak{A}$ iff for any $\emptyset \neq M \subseteq A$ that is maximally homogeneous for $\phi(\bar{c}, x, y)$ in $\mathfrak{A}, D_{A}(M, \phi(\bar{c}, x, y), 1,0)$ is dense in $P(A)$.

Lemma 2.2. Let $\mathfrak{A} \subseteq \mathcal{P}(\omega)$ be atomless, $\bar{c} \in A^{<\omega}, \phi(\bar{c}, x, y)$ qf and small in $\mathfrak{A}, e, f \in A$ and $M \neq \emptyset$ be maximally homogeneous for $\phi(\bar{c}, x, y)$ in $\mathfrak{A}$. Then $D_{A}(M, \phi(\bar{c}, x, y), e, f)$ is dense in $P(A)$ for any $e, f$ in $A$.

P r o of. [Ba-Ko, Lemmas 2.3 and 2.4].

Also the proof of the next lemma can be carried out as in [Ba-Ko]: just take a $u$ for $\mathfrak{A}$ and $\bar{M}$ in the same way as they take $x_{\alpha}$ for $\mathfrak{B}_{\alpha}$ and $M_{\alpha+1}$.

LEMma 2.3. Let $\mathfrak{A} \subseteq \mathcal{P}(\omega)$ be atomless and countable and let $\bar{M}$ be a countable subset of

$\left\{(M, \phi(\bar{c}, x, y)) \mid \bar{c} \in A^{<\omega}, \phi(\bar{c}, x, y) \in \mathcal{L}_{\omega \omega}\left[\tau_{B A}\right] q f, \phi(\bar{c}, x, y)\right.$ small in $A$ and $M$ is maximally homogeneous for $\phi(\bar{c}, x, y)$ in $A\}$.

Then for any $(a, b)_{A} \in P(A)$ there is a $u \in(a, b)_{\mathcal{P}(\omega)}$ such that:

1. $u \notin A$.

2. $[A \cup\{u\}]^{\mathcal{P}(\omega)}$, the subalgebra generated by $A \cup\{u\}$ in $\mathcal{P}(\omega)$, is atomless.

3. For any $(M, \phi(\bar{c}, x, y)) \in \bar{M}$ the set $M$ is maximally homogeneous for $\phi(\bar{c}, x, y)$ also in $[A \cup\{u\}]^{\mathcal{P}(\omega)}$.

Now using Lemma 2.3 and $\diamond$, we can construct our $\mathfrak{B}$. Let $\left\langle S_{\alpha} \mid \alpha \in \omega_{1}\right\rangle$ be a $\diamond$-sequence. Let $\left\langle a_{\xi} \mid \xi \in \omega_{1}\right\rangle$ be an enumeration of $\mathcal{P}(\omega)$ in which each element of $\mathcal{P}(\omega)$ appears $\omega_{1}$ times.

In step $\alpha+1$, let $M_{\alpha+1}=M_{\alpha} \cup\left\{\left(\left\{a_{\xi} \mid \xi \in S_{\alpha}\right\}, \phi(\bar{c}, x, y)\right) \mid\left\{a_{\xi} \mid \xi \in S_{\alpha}\right\}\right.$ is a maximally homogeneous set for $\phi(\bar{c}, x, y)$ in $\mathfrak{B}_{\alpha}$ and $\phi(\bar{c}, x, y)$ is small in $\mathfrak{B}_{\alpha}$ and $\left.\bar{c} \in B_{\alpha}\right\}$. Apply Lemma 2.3 with $\mathfrak{A}=\mathfrak{B}_{\alpha}$ and $\bar{M}=M_{\alpha+1}$ to get an $x_{\alpha}$. Define $B_{\alpha+1}$ as $\left[B_{\alpha} \cup\left\{x_{\alpha}\right\}\right]^{\mathcal{P}(\omega)}$. Let $\mathfrak{B}=\bigcup\left\{\mathfrak{B}_{\alpha} \mid \alpha \in \omega_{1}\right\}$. Take the $x_{\alpha}$ so that $\mathfrak{B} \vDash \forall x\left(x \neq 0 \rightarrow Q_{1} y y \subseteq x\right)$. Then it is easy to see that for any $\phi(\bar{c}, x, y)$ which is small in every $\mathfrak{B}_{\alpha}$ with $\bar{c} \in B_{\alpha}$, we have 
$\mathfrak{B} \vDash \neg Q_{1}^{2} x y \phi(\bar{c}, x, y)$. In particular, $\mathfrak{B}$ is a model of $\phi$ from the introduction (because " $x \nsubseteq y$ " is small), hence $\mathfrak{B}$ is rigid.

3. Large homogeneous sets. The aim of this section is to define a mapping

$$
\begin{aligned}
\operatorname{big}: \bigcup_{r \in \omega} \mathcal{L}_{\omega \omega}\left[\tau_{B A}\right](\stackrel{r}{z}, x, y) & \left.\rightarrow \bigcup_{r \in \omega} \mathcal{L}_{\omega \omega}\left[\tau_{B A}\right] \stackrel{r}{z}\right), \\
\phi(\stackrel{r}{z}, x, y) & \mapsto \operatorname{big}(\phi(\stackrel{r}{z}, x, y)) \stackrel{r}{z}),
\end{aligned}
$$

such that for every $\phi(\underset{z}{r}, x, y) \in \mathcal{L}_{\omega \omega}\left[\tau_{B A}\right]$

$$
\mathfrak{B} \vDash \forall z \stackrel{r}{z}\left(Q_{1}^{2} x y \phi(\stackrel{r}{z}, x, y) \leftrightarrow \operatorname{big}(\phi(\stackrel{r}{z}, x, y))(\stackrel{r}{z})\right) .
$$

Then $\Phi_{2}$ will be

$\{\phi(\bar{z}, x, y) \mid \operatorname{big}(\phi(\bar{z}, x, y))(\bar{z})$ is valid in any atomless Boolean algebra $\}$.

In order to simplify the notation we tacitly assume that always the variables $x$ and $y$ are intended to be quantified by $Q_{1}^{2}$.

Let $\mathfrak{A}$ be any atomless Boolean algebra. Since $\mathfrak{A}$ admits the elimination of $\exists$ it is enough to define big for quantifierfree $\phi(\stackrel{r}{z}, x, y) \in \mathcal{L}_{\omega \omega}\left[\tau_{B A}\right]$.

For any $\bar{c} \in A$ and qf $\phi(\bar{c}, x, y)$ there is a qf $\psi\left(\bar{c}^{\prime}, x, y\right)$ such that $\bar{c}^{\prime}$ is an (injective) enumeration of the atoms of the subalgebra generated by $\bar{c}$, and $\mathfrak{A} \vDash \forall x y\left(\psi\left(\bar{c}^{\prime}, x, y\right) \leftrightarrow \phi(\bar{c}, x, y)\right)$. Also if $\phi(\bar{z}, x, y)$ is a disjunction $\bigvee_{i}\left(\phi(\bar{z}, x, y) \wedge \psi_{i}(\bar{z})\right)$ then knowing $\chi_{i}=\operatorname{big}\left(\phi(\bar{z}, x, y) \wedge \psi_{i}(\bar{z})\right)(\bar{z})$ we can define $\operatorname{big}(\phi(\bar{z}, x, y))(\bar{z})$ to be $\bigvee_{i} \chi_{i}$. Hence it suffices to define $\operatorname{big}(\phi(\bar{z}, x, y))(\bar{z})$ only for those qf $\phi(\bar{z}, x, y)$ that imply that $\left\{z_{0}, \ldots, z_{r-1}\right\}$ is the set of atoms in the subalgebra generated by $\left\{z_{0}, \ldots, z_{r-1}\right\}$.

If $H$ is an uncountable homogeneous set for $\phi(\stackrel{r}{c}, x, y)$, then there is an $\mathcal{L}_{\omega \omega}$-1-type $t(\stackrel{r}{c}, x)$ over $\stackrel{r}{c}$ and an uncountable $H_{1} \subseteq H$ such that every element of $H_{1}$ has the $\mathcal{L}_{\omega \omega}$-1-type $\operatorname{tp}(x / \stackrel{r}{c})=t(\stackrel{r}{c}, x)$ over $\stackrel{r}{c}$. Hence it is enough to define big for the $\phi(\stackrel{r}{z}, x, y)$ with the above mentioned property and the additional property that there is an $\mathcal{L}_{\omega \omega}$-1-type $t(\stackrel{r}{z}, x)$ over $\underset{z}{z}$ (independent of the assignment $\stackrel{r}{c}$ of $\stackrel{r}{z}$, because we consider only $\stackrel{r}{c}$ that are atoms in the subalgebra generated by $\stackrel{r}{z}$ ) such that

$$
\mathfrak{A} \vDash \forall x y \stackrel{r}{z}(\phi(\stackrel{r}{z}, x, y) \leftrightarrow(\phi(\stackrel{r}{z}, x, y) \wedge t(\stackrel{r}{z}, x)=\operatorname{tp}(x / \stackrel{r}{z}) \wedge t(\stackrel{r}{z}, y)=\operatorname{tp}(y / \stackrel{r}{z}))) .
$$

We will call such formulas special. Finally, note that any $\mathcal{L}_{\omega \omega}$-2-type $t(\stackrel{r}{c}, x, y)$ over $\stackrel{r}{c}$ is determined by the corresponding $r$-tuple of the quantifierfree types of $x \cap c_{i}, y \cap c_{i}$ in $\left\{a \in A \mid a \subseteq c_{i}\right\}, i<r$. For any such type there are 15 possibilities, and under the condition $\operatorname{tp}(x / z)=\operatorname{tp}(y / z)$ there remain the 9 possibilities not marked with an $\bullet$ in the table below. 
The possibilities for the quantifierfree types of $x \cap c_{i}, y \cap c_{i}, i<r$, in $\left\{a \in A \mid a \subseteq c_{i}\right\}$

\begin{tabular}{|c|c|c|c|c|c|}
\hline No. & $x \cap y \cap z_{i}$ & $(-x) \cap(-y) \cap z_{i}$ & $x \cap(-y) \cap z_{i}$ & $(-x) \cap y \cap z_{i}$ & Remarks \\
\hline 0 & $\neq 0$ & $\neq 0$ & $\neq 0$ & $\neq 0$ & \\
\hline 1 & $\neq 0$ & $\neq \neq 0$ & $\neq 0$ & 0 & \\
\hline 2 & $\neq 0$ & $\neq 0$ & 0 & $\neq 0$ & \\
\hline 3 & $\neq 0$ & $\neq 0$ & 0 & 0 & $\begin{array}{c}x \cap z_{i}= \\
y \cap z_{i} \neq 0, z_{i}\end{array}$ \\
\hline 4 & $\neq 0$ & 0 & $\neq 0$ & $\neq 0$ & \\
\hline$\bullet 5$ & $\neq 0$ & 0 & $\neq 0$ & 0 & $\begin{array}{l}x \cap z_{i}=z_{i} \\
y \cap z_{i} \neq z_{i}\end{array}$ \\
\hline$\bullet 6$ & $\neq 0$ & 0 & 0 & $\neq 0$ & $\begin{array}{l}y \cap z_{i}=z_{i} \\
x \cap z_{i} \neq z_{i}\end{array}$ \\
\hline 7 & $\neq 0$ & 0 & 0 & 0 & $\begin{array}{c}x \cap z_{i}= \\
y \cap z_{i}=z_{i}\end{array}$ \\
\hline 8 & 0 & $\neq 0$ & $\neq 0$ & $\neq 0$ & \\
\hline$\bullet 9$ & 0 & $\neq 0$ & $\neq 0$ & 0 & $\begin{array}{l}x \cap z_{i} \neq 0 \\
y \cap z_{i}=0\end{array}$ \\
\hline$\bullet 10$ & 0 & $\neq 0$ & 0 & $\neq 0$ & $\begin{array}{l}x \cap z_{i}=0 \\
y \cap z_{i} \neq 0\end{array}$ \\
\hline 11 & 0 & $\neq 0$ & 0 & 0 & $\begin{array}{c}x \cap z_{i}= \\
y \cap z_{i}=0\end{array}$ \\
\hline 12 & 0 & 0 & $\neq 0$ & $\neq 0$ & $\begin{array}{c}x \cap z_{i} \neq=0, z_{i} \\
y \cap z_{i}=(-x) \cap z_{i}\end{array}$ \\
\hline$\bullet 13$ & 0 & 0 & $\neq 0$ & 0 & $\begin{array}{c}x \cap z_{i}=z_{i} \\
y \cap z_{i}=0\end{array}$ \\
\hline$\bullet 14$ & 0 & 0 & 0 & $\neq 0$ & $\begin{array}{c}x \cap z_{i}=0 \\
y \cap z_{i}=z_{i}\end{array}$ \\
\hline
\end{tabular}

Let $\phi^{k}\left(z_{i}, x \cap z_{i}, y \cap z_{i}\right)$ say "the $\mathcal{L}_{\omega \omega}$-type of $x \cap c_{i}, y \cap c_{i}$ over $c_{i}$ has number $k ", k=0, \ldots, 14$. The disjunction $\phi^{012}(u, v, w):=\phi^{0}(u, v, w) \vee$ $\phi^{1}(u, v, w) \vee \phi^{2}(u, v, w)$ will play an important role in the following.

Definition 3.1. Let $\phi(r z, x, y) \in \mathcal{L}_{\omega \omega}\left[\tau_{B A}\right]$ be quantifierfree and be of the special form as described above.

$$
\begin{aligned}
& \operatorname{big}(\phi(\stackrel{r}{z}, x, y))(\stackrel{r}{z})= \\
& \exists a \subset b \forall x y\left(\left(a \subseteq x, y \subseteq b \wedge \bigwedge_{i<r}\left((b \backslash a) \cap z_{i} \neq 0 \rightarrow \phi^{012}\left(z_{i}, x \cap z_{i}, y \cap z_{i}\right)\right)\right)\right. \\
&\rightarrow \phi(z, x, y, y)) .
\end{aligned}
$$

Equivalent to $\operatorname{big}(\phi(\stackrel{r}{z}, x, y))(\stackrel{r}{z})$ is the formula

$$
\begin{aligned}
\bigvee_{I_{0} \cup \dot{\cup} I_{1} \cup I_{2} \cup \dot{\cup} I_{3}=\{0, \ldots, r-1\}, I_{0} \neq 0} \forall x y & \left(\bigwedge_{i \in I_{0}} \phi^{012}\left(z_{i}, x \cap z_{i}, y \cap z_{i}\right)\right. \\
& \wedge \bigwedge_{i \in I_{1}} x \cap z_{i}=y \cap z_{i} \neq 0, z_{i}
\end{aligned}
$$




$$
\begin{aligned}
& \wedge \bigwedge_{i \in I_{2}} x \cap z_{i}=y \cap z_{i}=0 \\
& \left.\left.\wedge \bigwedge_{i \in I_{3}} x \cap z_{i}=y \cap z_{i}=z_{i}\right) \rightarrow \phi(\stackrel{r}{z}, x, y)\right),
\end{aligned}
$$

$(\dot{\cup}$ denotes the disjoint union) which will be useful for the easy direction of $(*)$ :

Lemma 3.2. Let $\mathfrak{A}$ be an atomless Boolean algebra. Let $\mathfrak{A} \vDash \forall x \neq 0$ $Q_{1} y y \subseteq x$, and $\phi(\stackrel{r}{z}, x, y)$ be as above. Then $\mathfrak{A} \vDash \forall z(\operatorname{big}(\phi(\stackrel{r}{z}, x, y))(\stackrel{r}{z}) \rightarrow$ $\left.Q_{1}^{2} x y \phi(\stackrel{r}{z}, x, y)\right)$.

Proof. Let $\mathfrak{A} \vDash \operatorname{big}(\phi(\stackrel{r}{z}, x, y))(\stackrel{r}{c})$. For $i \in I_{0}$ take an uncountable set $H_{i} \subseteq\left(0, c_{i}\right)_{\mathfrak{A}}$ such that for any $x \in H_{i}$ the relative complement $c_{i} \backslash x \notin H_{i}$. Let $\left\langle h_{i, \alpha} \mid \alpha \in \omega_{1}\right\rangle$ be an injective enumeration of a subset of $H_{i}$. Finally, for $i \in I_{1}$ let $H_{i}=\left\{d_{i}\right\}$ for some $d_{i}$ with $0 \subset d_{i} \subset c_{i}$, for $i \in I_{2}$ let $H_{i}=\{0\}$, and for $i \in I_{3}$ let $H_{i}=\left\{c_{i}\right\}$. Then

$$
H:=\left\{\bigcup\left\{h_{i, \alpha} \mid i \in I_{0}\right\} \cup \bigcup\left\{d_{i} \mid i \in I_{1}\right\} \cup \bigcup\left\{c_{i} \mid i \in I_{3}\right\} \mid \alpha \in \omega_{1}\right\}
$$

is an uncountable homogeneous set for $\phi(\stackrel{r}{c}, x, y)$.

Now for $\mathfrak{B}$ as in Section 2, we shall prove the other direction of $(*)$. By the construction, it would suffice to show:

(**) For any enumeration $\stackrel{r}{c}$ of the atoms in the subalgebra of $\mathfrak{B}$ generated by $\stackrel{r}{z}$, if $\mathfrak{B} \vDash \neg \operatorname{big}(\phi(\underset{z}{z}, x, y))(\stackrel{r}{c})$, then $\phi(\stackrel{r}{c}, x, y)$ is small in every $\mathfrak{B}_{\alpha}$ with $\stackrel{r}{c} \in B_{\alpha}$.

Unfortunately, this is true only for $\phi(\stackrel{r}{c}, x, y)$ that do not forbid certain equalities of Boolean terms. We introduce some notation and then give a sketch of our proof of the hard direction of $(*)$.

We say briefly " $\phi(\stackrel{r}{z}, x, y)$ is valid" or just " $\phi$ " for " $\phi(\underset{z}{z}, x, y)$ is valid in all atomless Boolean algebras if the assignment of $\underset{z}{r}$ is an enumeration of the atoms in the subalgebra generated by $\stackrel{r}{z} " . \phi(\underset{z}{z}, x, y)$ is satisfiable or consistent if $\neg \phi(\underset{z}{r}, x, y)$ is not valid.

For a given special $\phi(\stackrel{r}{z}, x, y)$ set

$$
R(\phi):=\left\{i<r \mid \phi \rightarrow x \cap z_{i}=y \cap z_{i} \text { is not valid }\right\} .
$$

We will define two mappings $s$ and enl from the set of all special $\phi(z, x, y)$ into itself. The mapping $s$ is a technical means used to prove enl $(\operatorname{enl}(s(\phi)))$ $\rightarrow \operatorname{enl}(s(\phi))$ (Lemma 3.7) and $\neg \operatorname{big}(s(\phi)) \rightarrow \neg \operatorname{big}(\operatorname{enl}(s(\phi)))$ (Lemma 3.8). Lemma 3.9 says that $(* *)$ is true for formulas of the form enl $(s(\phi))$ for some 
special $\phi$. Hence we get from the construction and from 3.8

$$
\mathfrak{B} \vDash \neg \operatorname{big}(s(\phi))(\stackrel{r}{c}) \rightarrow \neg Q_{1}^{2} x y \operatorname{enl}(s(\phi))(\stackrel{r}{c}, x, y),
$$

whence $s(\phi) \rightarrow \operatorname{enl}(s(\phi))$ and the monotonicity of the quantifier $Q_{1}^{2}$ imply

$$
\mathfrak{B} \vDash \neg \operatorname{big}(s(\phi))(\stackrel{r}{c}) \rightarrow \neg Q_{1}^{2} x y s(\phi)(\stackrel{r}{c}, x, y)
$$

(Theorem 3.10). Using this result we prove by induction on $\operatorname{card}(R(\phi))$, simultaneously for all special formulas $\phi$,

$$
\mathfrak{B} \vDash \neg \operatorname{big}(\phi)(\stackrel{r}{c}) \rightarrow \neg Q_{1}^{2} x y \phi(\stackrel{r}{c}, x, y),
$$

which will finish the proof of $(*)$.

In order to simplify the notation, we often suppress the free variables

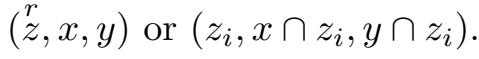

Definition 3.3 (The mapping $s$ ). For $R \subseteq r=\{0,1, \ldots, r-1\}$ and for $\chi\left(z_{i}, x \cap z_{i}, y \cap z_{i}\right) \in \mathcal{L}_{\omega \omega}\left[\tau_{B A}\right]$ we define

$$
s_{R}\left(\chi\left(z_{i}, x \cap z_{i}, y \cap z_{i}\right)\right):=\left\{\begin{array}{l}
\chi\left(z_{i}, x \cap z_{i}, y \cap z_{i}\right) \quad \text { if } i \notin R \text { or } \\
\phi^{012}\left(z_{i}, x \cap z_{i}, y \cap z_{i}\right) \rightarrow \chi\left(z_{i}, x \cap z_{i}, y \cap z_{i}\right) \\
\text { is valid; } \\
\chi\left(z_{i}, x \cap z_{i}, y \cap z_{i}\right) \wedge x \cap z_{i} \neq y \cap z_{i} \\
\text { else. }
\end{array}\right.
$$

Let $S=\left\{\bigwedge_{i<r} \chi_{w, i}\left(z_{i}, x \cap z_{i}, y \cap z_{i}\right) \mid w \in W\right\}$ be a finite set such that for all $w \in W$ the conjunction $\bigwedge_{i<r} \chi_{w, i}\left(z_{i}, x \cap z_{i}, y \cap z_{i}\right)$ is satisfiable and $\bigwedge_{i<r} \chi_{w, i}\left(z_{i}, x \cap z_{i}, y \cap z_{i}\right) \rightarrow \phi(\stackrel{r}{z}, x, y)$ is valid, and such that for any satisfiable conjunction $\delta=\bigwedge_{i<r} \chi_{i}^{\prime}\left(z_{i}, x \cap z_{i}, y \cap z_{i}\right)$ such that $\delta \rightarrow \phi(\stackrel{r}{z}, x, y)$ is valid there is a $w \in W$ with $\bigwedge_{i<r} \chi_{i}^{\prime}\left(z_{i}, x \cap z_{i}, y \cap z_{i}\right) \rightarrow \bigwedge_{i<r} \chi_{w, i}\left(z_{i}, x \cap z_{i}, y \cap z_{i}\right)$. We will call such a set $S$ a set of representatives for $\phi$. Given such a set, let $R=R(\phi)$ and define

$$
s(\phi(\stackrel{r}{z}, x, y))=\bigvee_{w \in W} \bigwedge_{i<r} s_{R}\left(\chi_{w, i}\left(z_{i}, x \cap z_{i}, y \cap z_{i}\right)\right) .
$$

If $\vDash \neg \exists x y \stackrel{r}{z} \phi(\stackrel{r}{z}, x, y)$, then let $s(\phi(\stackrel{r}{z}, x, y))$ be any inconsistent formula.

A brief reflection shows that $s(\phi)$ is well defined up to logical equivalence: Let $S^{\prime}=\left\{\bigwedge_{i<r} \chi_{w^{\prime}, i}^{\prime}\left(z_{i}, x \cap z_{i}, y \cap z_{i}\right) \mid w^{\prime} \in W^{\prime}\right\}$ be another set of representatives for $\phi$.

For $\bigvee_{w^{\prime} \in W^{\prime}} \bigwedge_{i<r} s_{R}\left(\chi_{w^{\prime}, i}^{\prime}\right) \rightarrow \bigvee_{w \in W} \bigwedge_{i<r} s_{R}\left(\chi_{w, i}\right)$, it suffices to show that for each $w^{\prime} \in W^{\prime}$ there is some $w \in W$ such that $\bigwedge_{i<r} s_{R}\left(\chi_{w^{\prime}, i}^{\prime}\right) \rightarrow$ $\bigwedge_{i<r} s_{R}\left(\chi_{w, i}\right)$. Let $w^{\prime} \in W^{\prime}$ be given. Since $S$ is a set of representatives for $\phi$ there is a $w \in W$ such that $\bigwedge_{i<r} \chi_{w^{\prime}, i}^{\prime} \rightarrow \bigwedge_{i<r} \chi_{w, i}$, which is equivalent to 
$\chi_{w^{\prime}, i}^{\prime} \rightarrow \chi_{w, i}$ for $i<r$. Immediately from the definition of $s_{R}$, if $\chi_{w^{\prime}, i}^{\prime} \rightarrow \chi_{w, i}$, then $s_{R}\left(\chi_{w^{\prime}, i}^{\prime}\right) \rightarrow s_{R}\left(\chi_{w, i}\right)$. Hence $\bigwedge_{i<r} s_{R}\left(\chi_{w^{\prime}, i}^{\prime}\right) \rightarrow \bigwedge_{i<r} s_{R}\left(\chi_{w, i}\right)$.

The other direction follows by symmetry.

R e m ark. $s(\phi)$ may be unsatisfiable, e.g. for $\phi=\left(x \cap z_{0}=y \cap z_{0} \wedge x \cap z_{1} \subset\right.$ $\left.y \cap z_{1}\right) \vee\left(x \cap z_{0} \subset y \cap z_{0} \wedge x \cap z_{1}=y \cap z_{1}\right) \wedge \bigwedge_{i=0,1} x \cap z_{i} \neq z_{i}, 0 \wedge \bigwedge_{i=0,1} y \cap z_{i} \neq$ $z_{i}, 0 \wedge z_{0} \cap z_{1}=0 \wedge z_{0} \cup z_{1}=1$.

Definition 3.4 (The mapping enl). For $\chi\left(z_{i}, x \cap z_{i}, y \cap z_{i}\right) \in \mathcal{L}_{\omega \omega}\left[\tau_{B A}\right]$ we define

$\operatorname{enl}\left(\chi\left(z_{i}, x \cap z_{i}, y \cap z_{i}\right)\right):=\left\{\begin{array}{l}\chi\left(z_{i}, x \cap z_{i}, y \cap z_{i}\right) \\ \vee\left(x \cap z_{i}=(-y) \cap z_{i} \wedge \exists x \chi\left(z_{i}, x \cap z_{i}, y \cap z_{i}\right)\right. \\ \left.\wedge \exists y \chi\left(z_{i}, x \cap z_{i}, y \cap z_{i}\right)\right) \\ \\ \text { if } \phi^{012}\left(z_{i}, x \cap z_{i}, y \cap z_{i}\right) \rightarrow \chi\left(z_{i}, x \cap z_{i}, y \cap z_{i}\right) \\ \text { is not valid; } \\ \chi\left(z_{i}, x \cap z_{i}, y \cap z_{i}\right) \vee\left(\left(x \cap z_{i}=(-y) \cap z_{i}\right.\right. \\ \left.\vee x \cap z_{i}=y \cap z_{i}\right) \wedge \exists x \chi\left(z_{i}, x \cap z_{i}, y \cap z_{i}\right) \\ \left.\wedge \exists y \chi\left(z_{i}, x \cap z_{i}, y \cap z_{i}\right)\right) \\ \text { otherwise. }\end{array}\right.$

Let $\left\{\bigwedge_{i<r} \chi_{w, i}\left(z_{i}, x \cap z_{i}, y \cap z_{i}\right) \mid w \in W\right\}$ be a set of representatives for $\phi$. Then set

$$
\operatorname{enl}(\phi(\stackrel{r}{z}, x, y))=\bigvee_{w \in W} \bigwedge_{i<r} \operatorname{enl}\left(\chi_{w, i}\left(z_{i}, x \cap z_{i}, y \cap z_{i}\right)\right)
$$

If $\vDash \neg \exists x y \stackrel{r}{z} \phi(\stackrel{r}{z}, x, y)$, then let $\operatorname{enl}(\phi(\stackrel{r}{z}, x, y))$ be any inconsistent formula.

From the fact that $\chi_{w^{\prime}, i}^{\prime} \rightarrow \chi_{w, i} \operatorname{implies} \operatorname{enl}\left(\chi_{w^{\prime}, i}^{\prime}\right) \rightarrow \operatorname{enl}\left(\chi_{w, i}\right)$, we conclude by an analogous consideration as above that $\operatorname{enl}(\phi)$ is well-defined.

In order to apply Lemmas 2.2 and 2.3 we may replace enl $(\phi(\underset{z}{r}, x, y))$ by an equivalent (with respect to the theory of atomless Boolean algebras) qf formula.

The next two lemmas collect some properties of $s$ and enl that will be useful in the proofs of 3.7 and of 3.8 .

LEMMA 3.5. Let $\chi_{s}\left(z_{i}, x \cap z_{i}, y \cap z_{i}\right), s=0,1$, be qf and $R \subseteq r$.

(i) $\left(\operatorname{enl}\left(\chi_{0}\right) \vee \operatorname{enl}\left(\chi_{1}\right)\right) \rightarrow \operatorname{enl}\left(\chi_{0} \vee \chi_{1}\right)$.

(ii) $\left(s_{R}\left(\chi_{0}\right) \vee s_{R}\left(\chi_{1}\right)\right) \rightarrow s_{R}\left(\chi_{0} \vee \chi_{1}\right)$.

For (iii), (iv) and (v), assume additionally that $\chi_{s}\left(z_{i}, x \cap z_{i}, y \cap z_{i}\right)$, $s=0,1$, determine the same 1-type $t\left(z_{i}, x \cap z_{i}\right)$ of $x \cap z_{i}$ over $z_{i}$ and of $y \cap z_{i}$ over $z_{i}$. 
(iii) Assume that, for $s=0,1$, if not $\phi^{012}\left(z_{i}, x \cap z_{i}, y \cap z_{i}\right) \rightarrow \chi_{s}\left(z_{i}, x \cap\right.$ $\left.z_{i}, y \cap z_{i}\right)$, then $\chi_{s}\left(z_{i}, x \cap z_{i}, y \cap z_{i}\right) \rightarrow x \cap z_{i} \neq y \cap z_{i}$. Then $\left(\operatorname{enl}\left(\chi_{0}\right) \wedge\right.$ $\left.\operatorname{enl}\left(\chi_{1}\right)\right) \rightarrow \operatorname{enl}\left(\chi_{0} \wedge \chi_{1}\right)$.

(iv) $\left(s_{R}\left(\chi_{0}\right) \wedge s_{R}\left(\chi_{1}\right)\right) \rightarrow s_{R}\left(\chi_{0} \wedge \chi_{1}\right)$.

(v) Assume that $\chi_{s} \rightarrow x \cap z_{i}=y \cap z_{i}$ for $s=0,1$ if $i \notin R$. Then for any $i<r$ the formula

$$
\begin{aligned}
\left(\operatorname{enl}\left(s_{R}\left(\chi_{0}\right)\right)\left(z_{i}, x \cap z_{i}, y \cap z_{i}\right) \wedge\right. & \left.\operatorname{enl}\left(s_{R}\left(\chi_{1}\right)\right)\left(z_{i}, x \cap z_{i}, y \cap z_{i}\right)\right) \\
& \rightarrow \operatorname{enl}\left(s_{R}\left(\chi_{0} \wedge \chi_{1}\right)\right)\left(z_{i}, x \cap z_{i}, y \cap z_{i}\right)
\end{aligned}
$$

is valid.

Proof. (i), (ii) $\chi_{s} \rightarrow \chi_{0} \vee \chi_{1}$ implies enl $\left(\chi_{s}\right) \rightarrow \operatorname{enl}\left(\chi_{0} \vee \chi_{1}\right)$ and $s_{R}\left(\chi_{s}\right) \rightarrow s_{R}\left(\chi_{0} \vee \chi_{1}\right)$.

(iii) Define

$\phi=\left(z_{i}, x \cap z_{i}, y \cap z_{i}\right):=x \cap z_{i}=y \cap z_{i} \wedge t\left(z_{i}, x \cap z_{i}\right) \quad$ and

$\phi_{-}\left(z_{i}, x \cap z_{i}, y \cap z_{i}\right):=x \cap z_{i}=(-y) \cap z_{i} \wedge t\left(z_{i}, x \cap z_{i}\right) \wedge t\left(z_{i}, y \cap z_{i}\right)$.

Case 1: $\phi^{012} \rightarrow \chi_{s}$ for $s=0,1$. Then $\phi^{012} \rightarrow \chi_{0} \wedge \chi_{1}$ and $\operatorname{enl}\left(\chi_{0}\right) \wedge$ $\operatorname{enl}\left(\chi_{1}\right)=\left(\chi_{0} \vee \phi_{-} \vee \phi_{=}\right) \wedge\left(\chi_{1} \vee \phi_{-} \vee \phi_{=}\right) \leftrightarrow\left(\chi_{0} \wedge \chi_{1}\right) \vee \phi_{-} \vee \phi_{=}=\operatorname{enl}\left(\chi_{0} \wedge \chi_{1}\right)$

Case 2: Not $\phi^{012} \rightarrow \chi_{s}$ for $s=0,1$. Then not $\phi^{012} \rightarrow \chi_{0} \wedge \chi_{1}$ and $\operatorname{enl}\left(\chi_{0}\right) \wedge \operatorname{enl}\left(\chi_{1}\right)=\left(\chi_{0} \vee \phi_{-}\right) \wedge\left(\chi_{1} \vee \phi_{-}\right) \leftrightarrow\left(\chi_{0} \wedge \chi_{1}\right) \vee \phi_{-}=\operatorname{enl}\left(\chi_{0} \wedge \chi_{1}\right)$

Case 3: $\phi^{012} \rightarrow \chi_{0}$ and not $\phi^{012} \rightarrow \chi_{1}$. Then not $\phi^{012} \rightarrow \chi_{0} \wedge \chi_{1}$ and $\operatorname{enl}\left(\chi_{0}\right) \wedge \operatorname{enl}\left(\chi_{1}\right)=\left(\chi_{0} \vee \phi_{-} \vee \phi_{=}\right) \wedge\left(\chi_{1} \vee \phi_{-}\right) \leftrightarrow\left(\chi_{0} \wedge \chi_{1}\right) \vee \phi_{-} \vee\left(\phi_{=} \wedge \chi_{1}\right)$ Since by the assumption of (iii), $\phi_{=} \wedge \chi_{1}$ is not satisfiable, the latter formula is equivalent to $\left(\chi_{0} \wedge \chi_{1}\right) \vee \phi_{-}=\operatorname{enl}\left(\chi_{0} \wedge \chi_{1}\right)$.

(iv) Assume $i \in R$, otherwise $s_{R}$ does not change $\chi_{0}, \chi_{1}, \chi_{0} \wedge \chi_{1}$.

Case 1: $\phi^{012} \rightarrow \chi_{s}$ for $s=0,1$. Then $\phi^{012} \rightarrow \chi_{0} \wedge \chi_{1}$ and $s_{R}\left(\chi_{0}\right) \wedge$ $s_{R}\left(\chi_{1}\right)=\chi_{0} \wedge \chi_{1}=s_{R}\left(\chi_{0} \wedge \chi_{1}\right)$.

Case 2: E.g. not $\phi^{012} \rightarrow \chi_{0}$. Then not $\phi^{012} \rightarrow \chi_{0} \wedge \chi_{1}$ and $s_{R}\left(\chi_{0}\right) \wedge$ $s_{R}\left(\chi_{1}\right)=\left(\chi_{0} \wedge x \cap z_{i} \neq y \cap z_{i}\right) \wedge s_{R}\left(\chi_{1}\right) \leftrightarrow\left(\chi_{0} \wedge \chi_{1}\right) \wedge x \cap z_{i} \neq y \cap z_{i}=$ $s_{R}\left(\chi_{0} \wedge \chi_{1}\right)$.

(v) For $i \in R$, the assumptions for (iii) are true for $\psi_{s}=s_{R}\left(\chi_{s}\right)$. Hence by (iii) and (iv),

$$
\begin{aligned}
\left(\operatorname{enl}\left(s_{R}\left(\chi_{0}\right)\right)\left(z_{i}, x \cap z_{i}, y \cap z_{i}\right) \wedge\right. & \left.\operatorname{enl}\left(s_{R}\left(\chi_{1}\right)\right)\left(z_{i}, x \cap z_{i}, y \cap z_{i}\right)\right) \\
& \rightarrow \operatorname{enl}\left(s_{R}\left(\chi_{0} \wedge \chi_{1}\right)\right)\left(z_{i}, x \cap z_{i}, y \cap z_{i}\right) .
\end{aligned}
$$

For $i \notin R$, we have $\chi_{s} \rightarrow x \cap z_{i}=y \cap z_{i}$ for $s=0,1$ and hence $\operatorname{enl}\left(s_{R}\left(\chi_{0}\right)\right) \wedge$ $\operatorname{enl}\left(s_{R}\left(\chi_{1}\right)\right)=\left(\chi_{0} \vee \phi_{-}\right) \wedge\left(\chi_{1} \vee \phi_{-}\right) \leftrightarrow\left(\chi_{0} \wedge \chi_{1}\right) \vee \phi_{-}=\operatorname{enl}\left(s_{R}\left(\chi_{0} \wedge \chi_{1}\right)\right)$

LEMMA 3.6. Let $\phi$ be special and satisfiable, $R=R(\phi)$, and let $\left\{\bigwedge_{i<r} \chi_{w, i} \mid w \in W\right\}$ be a set of representatives for $\phi$.

(i) For any $\bigwedge_{i<r} \chi_{i}^{\prime} \rightarrow \bigvee_{w \in W} \bigwedge_{i<r} s_{R}\left(\chi_{w, i}\right)$, there is a $w \in W$ such that $\bigwedge_{i<r} \chi_{i}^{\prime} \rightarrow \bigwedge_{i<r} s_{R}\left(\chi_{w, i}\right)$. 
(ii) $\operatorname{enl}(s(\phi)) \leftrightarrow \bigvee_{w \in W} \bigwedge_{i<r} \operatorname{enl}\left(s_{R}\left(\chi_{w, i}\right)\right)$.

(iii) For any $\bigwedge_{i<r} \chi_{i}^{\prime} \rightarrow \bigvee_{w \in W} \bigwedge_{i<r} \operatorname{enl}\left(s_{R}\left(\chi_{w, i}\right)\right)$, there is a $w \in W$ such that $\bigwedge_{i<r} \chi_{i}^{\prime} \rightarrow \bigwedge_{i<r} \operatorname{enl}\left(s_{R}\left(\chi_{w, i}\right)\right)$.

Proof. We will first prove (iii). Then the proof of (i) which is similar but easier will be clear. Let $\bigwedge_{i<r} \chi_{i}^{\prime}\left(z_{i}, x \cap z_{i}, y \cap z_{i}\right)$ be consistent, otherwise one can take any $w \in W$.

For $i<r$ there is an $n_{i}, 0<n_{i}<15$, and there are $\widehat{\chi}_{i, 0}, \ldots, \widehat{\chi}_{i, n_{i}-1} \in$ $\left\{\phi^{0}, \ldots, \phi^{14}\right\}$ such that

$$
\bigwedge_{i<r} \chi_{i}^{\prime}\left(z_{i}, x \cap z_{i}, y \cap z_{i}\right) \leftrightarrow \bigwedge_{i<r}\left(\widehat{\chi}_{i, 0} \vee \ldots \vee \widehat{\chi}_{i, n_{i}-1}\right)\left(z_{i}, x \cap z_{i}, y \cap z_{i}\right) .
$$

We will show the claim by induction on $\prod_{i<r} n_{i}$.

C a s e $\prod_{i<r} n_{i}=1$. Take an atomless Boolean algebra $\mathfrak{A}$ and ${ }_{c}^{r} \in A$ such that $\stackrel{r}{c}$ is an enumeration of all the atoms in the generated subalgebra. Take $a, b \in A$ such that $\mathfrak{A} \vDash \bigwedge_{i<r} \chi_{i}^{\prime}\left(c_{i}, a \cap c_{i}, b \cap c_{i}\right)$. Then there is some $w \in W$ with $\mathfrak{A} \vDash \bigwedge_{i<r} \operatorname{enl}\left(s_{R}\left(\chi_{w, i}\left(c_{i}, a \cap c_{i}, b \cap c_{i}\right)\right)\right)$. Since $\bigwedge_{i<r} \chi_{i}^{\prime}\left(z_{i}, x \cap z_{i}, y \cap z_{i}\right)$ defines an $\mathcal{L}_{\omega \omega}$-2-type of $(x, y)$ over $\stackrel{r}{z}$, we have $\bigwedge_{i<r} \chi_{i}^{\prime}\left(z_{i}, x \cap z_{i}, y \cap z_{i}\right) \rightarrow$ $\bigwedge_{i<r} \operatorname{enl}\left(s_{R}\left(\chi_{w, i}\left(z_{i}, x \cap z_{i}, y \cap z_{i}\right)\right)\right)$.

Induction step. We consider the step from $\prod_{i<r} n_{i}$ to $\left(n_{0}+1\right)$ $\times \prod_{0<i<r} n_{i}$, the other cases are similar.

$$
\left(\widehat{\chi}_{0,0} \vee \ldots \vee \widehat{\chi}_{0, n_{0}}\right) \wedge \bigwedge_{0<i<r} \chi_{i}^{\prime} \leftrightarrow\left(\widehat{\chi}_{0,0} \wedge \bigwedge_{0<i<r} \chi_{i}^{\prime}\right) \vee\left(\left(\widehat{\chi}_{0,1} \vee \ldots \vee \widehat{\chi}_{0, n_{0}}\right) \wedge \bigwedge_{0<i<r} \chi_{i}^{\prime}\right) .
$$

By induction hypothesis there are $w^{\prime}, w^{\prime \prime} \in W$ such that

$$
\begin{aligned}
\widehat{\chi}_{0,0} & \wedge \bigwedge_{0<i<r} \chi_{i}^{\prime} \rightarrow \bigwedge_{i<r} \operatorname{enl}\left(s_{R}\left(\chi_{w^{\prime}, i}\right)\right), \\
\left(\widehat{\chi}_{0,1} \vee \ldots \vee \widehat{\chi}_{0, n_{0}}\right) \wedge \bigwedge_{0<i<r} \chi_{i}^{\prime} & \rightarrow \bigwedge_{i<r} \operatorname{enl}\left(s_{R}\left(\chi_{w^{\prime \prime}, i}\right)\right) .
\end{aligned}
$$

Thus we have

$$
\begin{aligned}
& \left(\left(\widehat{\chi}_{0,0} \wedge \bigwedge_{0<i<r} \chi_{i}^{\prime}\right) \vee\left(\left(\widehat{\chi}_{0,1} \vee \ldots \vee \widehat{\chi}_{0, n_{0}}\right) \wedge \bigwedge_{0<i<r} \chi_{i}^{\prime}\right)\right) \rightarrow \\
& \quad\left(\operatorname{enl}\left(s_{R}\left(\chi_{w^{\prime}, 0}\right)\right) \vee \operatorname{enl}\left(s_{R}\left(\chi_{w^{\prime \prime}, 0}\right)\right)\right) \wedge \bigwedge_{0<i<r}\left(\operatorname{enl}\left(s_{R}\left(\chi_{w^{\prime}, i}\right)\right) \wedge \operatorname{enl}\left(s_{R}\left(\chi_{w^{\prime \prime}, i}\right)\right)\right) .
\end{aligned}
$$

Note that in the last conjunction we get "and" and not only "or", because

$$
\bigwedge_{0<i<r} \chi_{i}^{\prime} \rightarrow \bigwedge_{0<i<r} \operatorname{enl}\left(s_{R}\left(\chi_{w^{\prime}, i}\right)\right) \wedge \bigwedge_{0<i<r} \operatorname{enl}\left(s_{R}\left(\chi_{w^{\prime \prime}, i}\right)\right),
$$

as the situation below any $z_{i}$ is independent of the situation below the other $z_{j}$. 
From 3.5(i), (ii) and (v) we get

$$
\begin{aligned}
& \left(\widehat{\chi}_{0,0} \wedge \bigwedge_{0<i<r} \chi_{i}^{\prime}\right) \vee\left(\left(\widehat{\chi}_{0,1} \vee \ldots \vee \widehat{\chi}_{0, n_{0}}\right) \wedge \bigwedge_{0<i<r} \chi_{i}^{\prime}\right) \\
& \rightarrow \operatorname{enl}\left(s_{R}\left(\chi_{w^{\prime}, 0} \vee \chi_{w^{\prime \prime}, 0}\right)\right) \wedge \bigwedge_{0<i<r} \operatorname{enl}\left(s_{R}\left(\chi_{w^{\prime}, i} \wedge \chi_{w^{\prime \prime}, i}\right)\right) .
\end{aligned}
$$

Since $\left\{\bigwedge_{i<r} \chi_{w, i}\left(z_{i}, x \cap z_{i}, y \cap z_{i}\right) \mid w \in W\right\}$ is a set of representatives for $\phi(\stackrel{r}{z}, x, y)$ and since $w^{\prime}, w^{\prime \prime} \in W$, we have $\left(\chi_{w^{\prime}, 0} \vee \chi_{w^{\prime \prime}, 0}\right) \wedge \bigwedge_{0<i<r}\left(\chi_{w^{\prime}, i} \wedge\right.$ $\left.\chi_{w^{\prime \prime}, i}\right) \rightarrow \phi$ and there is a $w \in W$ such that

$$
\left(\chi_{w^{\prime}, 0} \vee \chi_{w^{\prime \prime}, 0}\right) \wedge \bigwedge_{0<i<r}\left(\chi_{w^{\prime}, i} \wedge \chi_{w^{\prime \prime}, i}\right) \rightarrow \bigwedge_{i<r} \chi_{w, i}
$$

For such a $w$ we have

$$
\operatorname{enl}\left(s_{R}\left(\chi_{w^{\prime}, 0} \vee \chi_{w^{\prime \prime}, 0}\right)\right) \wedge \bigwedge_{0<i<r} \operatorname{enl}\left(s_{R}\left(\chi_{w^{\prime}, i} \wedge \chi_{w^{\prime \prime}, i}\right)\right) \rightarrow \bigwedge_{i<r} \operatorname{enl}\left(s_{R}\left(\chi_{w, i}\right)\right),
$$

and thus the induction step is complete and (iii) is shown.

(ii) Assume $s(\phi)$ is satisfiable, otherwise both sides are not satisfiable. Let $S=\left\{\bigwedge_{i<r} \chi_{w, i} \mid w \in W\right\}$ be a set of representatives for $\phi$, and $S^{\prime}=$ $\left\{\bigwedge_{i<r} \chi_{w^{\prime}, i}^{\prime} \mid w^{\prime} \in W^{\prime}\right\}$ be a set of representatives for $s(\phi)=\bigvee_{w \in W} \bigwedge_{i<r}$ $s_{R}\left(\chi_{w, i}\right)$ such that $W^{\prime} \supseteq \widehat{W}:=\left\{w \in W \mid \bigwedge_{i<r} s_{R}\left(\chi_{w, i}\right)\right.$ is satisfiable $\}$ and $\chi_{w^{\prime}, i}^{\prime}=s_{R}\left(\chi_{w, i}\right)$ for $w \in \widehat{W}$.

By definition, $\operatorname{enl}\left(s_{R}(\phi)\right)=\bigvee_{w^{\prime} \in W^{\prime}} \bigwedge_{i<r} \operatorname{enl}\left(\chi_{w^{\prime}, i}^{\prime}\right)$. By (i), for any $w^{\prime} \in W^{\prime}$ there is some $w \in W$ such that $\bigwedge_{i<r} \chi_{w^{\prime}, i}^{\prime} \rightarrow \bigwedge_{i<r} s_{R}\left(\chi_{w, i}\right)$ and hence $\bigwedge_{i<r} \operatorname{enl}\left(\chi_{w^{\prime}, i}^{\prime}\right) \rightarrow \bigwedge_{i<r} \operatorname{enl}\left(s_{R}\left(\chi_{w, i}\right)\right)$. Thus enl $(s(\phi)) \rightarrow \bigvee_{w \in W} \bigwedge_{i<r}$ $\operatorname{enl}\left(s_{R}\left(\chi_{w, i}\right)\right)$. The other direction follows immediately from the choice of $S^{\prime}$ and the definition of enl.

Lemma 3.7. Let $\phi$ be a special formula. Then $\operatorname{enl}(\operatorname{enl}(s(\phi))) \leftrightarrow \operatorname{enl}(s(\phi))$.

Proof. Assume $s(\phi)$ is satisfiable, otherwise both sides are not satisfiable. Let $S, W$ be as above and $S^{\prime \prime}=\left\{\bigwedge_{i<r} \chi_{w^{\prime \prime}, i}^{\prime \prime} \mid w^{\prime \prime} \in W^{\prime \prime}\right\}$ be a set of representatives for enl $(s(\phi))$. By definition, enl $(\operatorname{enl}(s(\phi)))=\bigvee_{w^{\prime \prime} \in W^{\prime \prime}} \bigwedge_{i<r}$ $\operatorname{enl}\left(\chi_{w^{\prime \prime}, i}^{\prime \prime}\right)$. For $w^{\prime \prime} \in W^{\prime \prime}$ we have $\bigwedge_{i<r} \chi_{w^{\prime \prime}, i}^{\prime \prime} \rightarrow \operatorname{enl}(s(\phi))$, hence by 3.6(ii), $\bigwedge_{i<r} \chi_{w^{\prime \prime}, i}^{\prime \prime} \rightarrow \bigvee_{w \in W} \bigwedge_{i<r} \operatorname{enl}\left(s_{R}\left(\chi_{w, i}\right)\right)$. By 3.6(iii) there is some $w \in$ $W$ such that $\bigwedge_{i<r} \chi_{w^{\prime \prime}, i}^{\prime \prime} \rightarrow \bigwedge_{i<r} \operatorname{enl}\left(s_{R}\left(\chi_{w, i}\right)\right)$, whence $\bigwedge_{i<r} \operatorname{enl}\left(\chi_{w^{\prime \prime}, i}^{\prime \prime}\right) \rightarrow$ $\bigwedge_{i<r} \operatorname{enl}\left(\operatorname{enl}\left(s_{R}\left(\chi_{w, i}\right)\right)\right)$. It is easy to check that for qf $\chi\left(z_{i}, x \cap z_{i}, y \cap z_{i}\right)$ by definition

$$
\operatorname{enl}\left(\operatorname{enl}\left(\chi\left(z_{i}, x \cap z_{i}, y \cap z_{i}\right)\right)\right) \rightarrow \operatorname{enl}\left(\chi\left(z_{i}, x \cap z_{i}, y \cap z_{i}\right)\right) .
$$

Therefore $\bigwedge_{i<r} \operatorname{enl}\left(\chi_{w^{\prime \prime}, i}^{\prime \prime}\right) \rightarrow \bigwedge_{i<r} \operatorname{enl}\left(s_{R}\left(\chi_{w, i}\right)\right)$, and putting things together yields $\bigvee_{w^{\prime \prime} \in W^{\prime \prime}} \bigwedge_{i<r} \operatorname{enl}\left(\chi_{w^{\prime \prime}, i}^{\prime \prime}\right) \rightarrow \bigvee_{w \in W} \bigwedge_{i<r} \operatorname{enl}\left(s_{R}\left(\chi_{w, i}\right)\right)$, and, by 3.6(ii), $\bigvee_{w^{\prime \prime} \in W^{\prime \prime}} \bigwedge_{i<r} \operatorname{enl}\left(\chi_{w^{\prime \prime}, i}^{\prime \prime}\right) \rightarrow \operatorname{enl}(s(\phi))$. 
The other direction is obvious.

LEMMA 3.8. $\neg \operatorname{big}(s(\phi)) \rightarrow \neg \operatorname{big}(\operatorname{enl}(s(\phi)))$ is valid for special $\phi$.

Proof. Let $\mathfrak{A}$ be any atomless Boolean algebra. Assume $\mathfrak{A} \vDash$ $\operatorname{big}(\operatorname{enl}(s(\phi(\stackrel{r}{z}, x, y))))(\stackrel{r}{c})$. We show that $\mathfrak{A} \vDash \operatorname{big}(s(\phi(\stackrel{r}{z}, x, y)))(\stackrel{r}{c})$. Since the 1-types of $x$ and of $y$ over $\stackrel{r}{c}$ are determined by $\mathfrak{A} \vDash \exists y \operatorname{enl}(s(\phi(\stackrel{r}{c}, x, y)))$ and $\mathfrak{A} \vDash \exists x \operatorname{enl}(s(\phi(\stackrel{r}{c}, x, y)))$, there is just one pair $\left(I_{2}, I_{3}\right)$ such that

$$
\begin{aligned}
& \mathfrak{A} \vDash \bigvee_{\left\{\left(I_{0}, I_{1}\right) \mid I_{0} \dot{\cup} I_{1} \dot{\cup} I_{2} \dot{\cup} I_{3}=\{0, \ldots, r-1\}, I_{0} \neq 0\right\}} \forall x y \\
& \left(\left(\bigwedge_{i \in I_{0}} \phi^{012}\left(c_{i}, x \cap c_{i}, y \cap c_{i}\right) \wedge \bigwedge_{i \in I_{1}} x \cap c_{i}=y \cap c_{i} \neq 0, c_{i}\right.\right. \\
& \left.\left.\wedge \bigwedge_{i \in I_{2}} x \cap c_{i}=y \cap c_{i}=0 \wedge \bigwedge_{i \in I_{3}} x \cap c_{i}=y \cap c_{i}=c_{i}\right) \rightarrow \operatorname{enl}(s(\phi(\stackrel{r}{c}, x, y)))\right) .
\end{aligned}
$$

Take $I_{0} \subseteq$-maximal such that

$$
\begin{aligned}
\mathfrak{A} & \vDash \forall x y\left(\left(\bigwedge_{i \in I_{0}} \phi^{012}\left(c_{i}, x \cap c_{i}, y \cap c_{i}\right) \wedge \bigwedge_{i \in I_{1}} x \cap c_{i}=y \cap c_{i} \neq 0, c_{i}\right.\right. \\
& \left.\left.\wedge \bigwedge_{i \in I_{2}} x \cap c_{i}=y \cap c_{i}=0 \wedge \bigwedge_{i \in I_{3}} x \cap c_{i}=y \cap c_{i}=c_{i}\right) \rightarrow \operatorname{enl}(s(\phi(r, x, y)))\right) .
\end{aligned}
$$

Let $R=R(\phi)$ and $\left\{\bigwedge_{i<r} \chi_{w, i} \mid w \in W\right\}$ be a set of representatives for $\phi$. By 3.6(ii) and (iii) there is a $w \in W$ such that

$$
\begin{aligned}
\mathfrak{A} \vDash & \forall x y\left(\bigwedge_{i \in I_{0}} \phi^{012}\left(c_{i}, x \cap c_{i}, y \cap c_{i}\right) \wedge \bigwedge_{i \in I_{1}} x \cap c_{i}=y \cap c_{i} \neq 0, c_{i}\right. \\
& \left.\wedge \bigwedge_{i \in I_{2}} x \cap c_{i}=y \cap c_{i}=0 \wedge \bigwedge_{i \in I_{3}} x \cap c_{i}=y \cap c_{i}=c_{i}\right) \\
& \left.\rightarrow \bigwedge_{i<r} \operatorname{enl}\left(s_{R}\left(\chi_{w, i}\left(c_{i}, x \cap c_{i}, y \cap c_{i}\right)\right)\right)\right) .
\end{aligned}
$$

We claim that also

$$
\begin{aligned}
\mathfrak{A} \vDash & \forall x y\left(\bigwedge_{i \in I_{0}} \phi^{012}\left(c_{i}, x \cap c_{i}, y \cap c_{i}\right) \wedge \bigwedge_{i \in I_{1}} x \cap c_{i}=y \cap c_{i} \neq 0, c_{i}\right. \\
& \left.\wedge \bigwedge_{i \in I_{2}} x \cap c_{i}=y \cap c_{i}=0 \wedge \bigwedge_{i \in I_{3}} x \cap c_{i}=y \cap c_{i}=c_{i}\right) \\
& \left.\rightarrow \bigwedge_{i<r} s_{R}\left(\chi_{w, i}\left(c_{i}, x \cap c_{i}, y \cap c_{i}\right)\right)\right) .
\end{aligned}
$$

Indeed, by the definition of enl we have for any $s_{R}\left(\chi_{w, i}\left(z_{i}, x \cap z_{i}, y \cap z_{i}\right)\right)$ : For $i \in I_{0}$, if $\phi^{012} \rightarrow \operatorname{enl}\left(s_{R}\left(\chi_{w, i}\right)\right)$, then $\phi^{012} \rightarrow s_{R}\left(\chi_{w, i}\right)$. For $i \in I_{2}$, if $x \cap z_{i}=y \cap z_{i}=0 \rightarrow \operatorname{enl}\left(s_{R}\left(\chi_{w, i}\right)\right)$, then $x \cap z_{i}=y \cap z_{i}=0 \rightarrow s_{R}\left(\chi_{w, i}\right)$. 
For $i \in I_{3}$, if $x \cap z_{i}=y \cap z_{i}=z_{i} \rightarrow \operatorname{enl}\left(s_{R}\left(\chi_{w, i}\right)\right)$, then $x \cap z_{i}=y \cap z_{i}=$ $z_{i} \rightarrow s_{R}\left(\chi_{w, i}\right)$.

For $i \in I_{1}$ the formula $x \cap z_{i}=y \cap z_{i} \neq 0, z_{i} \wedge \operatorname{enl}\left(s_{R}\left(\chi_{w, i}\right)\right) \wedge \neg s_{R}\left(\chi_{w, i}\right)$ is consistent only if $\phi^{012} \rightarrow s_{R}\left(\chi_{w, i}\right)$. But then we could take $I_{0}^{\prime}:=I_{0} \cup\{i\}$ and $I_{1}^{\prime}=I_{1} \backslash\{i\}$ and replace $\left(I_{0}, I_{1}\right)$ by $\left(I_{0}^{\prime}, I_{1}^{\prime}\right)$, which contradicts the maximality of $I_{0}$.

Now we are ready to prove $(* *)$ for special formulas of the form $s(\phi)$.

LEMMA 3.9. Let $\phi$ be special and $\stackrel{r}{c} \in B$ be an $r$-tuple that consists of atoms in the generated subalgebra.

(i) If $\neg \operatorname{big}(\phi)$ and $\operatorname{enl}(\phi) \rightarrow \phi$ are valid, then for any $\alpha$ with $\stackrel{r}{c} \in B_{\alpha}$ the relation $\phi(\stackrel{r}{c}, x, y)$ is small in $\mathfrak{B}_{\alpha}$.

(ii) If $\neg \operatorname{big}(s(\phi))$ is valid, then for any $\alpha$ with $\stackrel{r}{c} \in B_{\alpha}$ the relation $\operatorname{enl}(s(\phi(\stackrel{r}{c}, x, y)))$ is small in $\mathfrak{B}_{\alpha}$.

Proof. (i) Let $\mathfrak{B} \vDash \neg \operatorname{big}(\phi(\stackrel{r}{z}, x, y))(\stackrel{r}{c})$ and $\stackrel{r}{c} \in B_{\alpha}$ be atoms in the generated subalgebra. Set $\mathfrak{B}_{\alpha}=: \mathfrak{A}$, and let $M \neq \emptyset$ be a maximally homogeneous set for $\phi(\stackrel{r}{c}, x, y)$ in $\mathfrak{A}$, and $(a, b)_{A} \in P(A)$, i.e. $(a, b)_{A}$ is an interval in $\mathfrak{A}$. Take $\left(a^{\prime}, b^{\prime}\right)_{A} \leq(a, b)_{A}$ such that there is just one $i \in r$, say $i_{0}$, with $\left(b^{\prime} \backslash a^{\prime}\right) \subseteq c_{i}$ and $c_{i} \cap a^{\prime} \neq 0$ and $b^{\prime} \cap c_{i} \neq c_{i}$. We assume $\mathfrak{B}$ (and also $\mathfrak{A}$ and $\mathcal{P}(\omega))$ satisfy

$$
\forall x \in\left(a^{\prime}, b^{\prime}\right)(\exists y \phi(\stackrel{r}{z}, x, y) \wedge \exists y \phi(\stackrel{r}{z}, y, x))(\stackrel{r}{c}),
$$

for otherwise $\left(a^{\prime}, b^{\prime}\right)_{A} \in D_{A}(M, \phi(\stackrel{r}{c}, x, y), 1,0)$.

Since $\mathfrak{B} \vDash \neg \operatorname{big}(\phi)(c)$, we have $\left(a^{\prime}, b^{\prime}\right)_{A} \cap M \neq\left(a^{\prime}, b^{\prime}\right)_{A}$. We fix a $d \in$ $\left(a^{\prime}, b^{\prime}\right)_{A} \backslash M$ and an $m \in M$ such that $\mathfrak{A} \vDash \neg \phi(\stackrel{r}{c}, d, m) \vee \neg \phi(\stackrel{r}{c}, m, d)$, say $\mathfrak{A} \vDash \neg \phi(c, d, m)$, and show that there is an $\left(a^{\prime \prime}, b^{\prime \prime}\right)_{A} \leq\left(a^{\prime}, b^{\prime}\right)_{A}$ such that for any $x \in\left(a^{\prime \prime}, b^{\prime \prime}\right)_{\mathcal{P}(\omega)}$ we have $x \in M$ or $\mathcal{P}(\omega) \vDash \neg \phi(\stackrel{r}{c}, x, m)$.

Then (i) will be proved, because such an $\left(a^{\prime \prime}, b^{\prime \prime}\right)_{A}$ is in $D_{A}(M, \phi(\stackrel{r}{c}, x, y)$, 1,0). Fix a set $\left\{\bigwedge_{i<r} \chi_{w, i} \mid w \in W\right\}$ of representatives for $\phi$.

Claim. $d \cap c_{i_{0}} \neq c_{i_{0}} \backslash m$.

Proof. $\phi(\stackrel{r}{z}, x, y)=\bigvee_{w \in W} \bigwedge_{i<r} \chi_{w, i}\left(z_{i}, x \cap z_{i}, y \cap z_{i}\right)$, w.l.o.g. $W=$ $\{0,1, \ldots, s-1\}$. Hence $\mathfrak{A} \vDash \bigwedge_{w \in W} \bigvee_{i<r} \neg \chi_{w, i}\left(c_{i}, d \cap c_{i}, m \cap c_{i}\right)$, say for $w=0,1, \ldots, s^{\prime}-1$

$$
\mathfrak{A} \vDash \bigvee_{i<r, i \neq i_{0}} \neg \chi_{w, i}\left(c_{i}, d \cap c_{i}, m \cap c_{i}\right),
$$

and for $w=s^{\prime}, s^{\prime}+1, \ldots, s-1$

$$
\mathfrak{A} \vDash \bigwedge_{i<r, i \neq i_{0}} \chi_{w, i}\left(c_{i}, d \cap c_{i}, m \cap c_{i}\right) \wedge \neg \chi_{w, i_{0}}\left(c_{i_{0}}, d \cap c_{i_{0}}, m \cap c_{i_{0}}\right) .
$$


We may assume $s>0$ and $s^{\prime} \leq s-1$, because otherwise $\left(a^{\prime}, b^{\prime}\right)_{A} \in$ $D_{A}(M, \phi(\stackrel{r}{c}, x, y), 1,0)$. Since

$$
\begin{aligned}
\mathfrak{A} F \forall x y\left(\left(\bigwedge_{s^{\prime} \leq w<s}\right.\right. & \bigwedge_{i<r, i \neq i_{0}} \chi_{w, i}\left(c_{i}, x \cap c_{i}, y \cap c_{i}\right) \\
& \left.\left.\wedge \bigvee_{s^{\prime} \leq w<s} \chi_{w, i_{0}}\left(c_{i_{0}}, x \cap c_{i_{0}}, y \cap c_{i_{0}}\right)\right) \rightarrow \phi(\stackrel{r}{c}, y, x)\right),
\end{aligned}
$$

we have

$$
\begin{aligned}
\mathfrak{A} \vDash \forall & \forall y\left(\bigwedge_{s^{\prime} \leq w<s} \bigwedge_{i<r, i \neq i_{0}} \chi_{w, i}\left(c_{i}, x \cap c_{i}, y \cap c_{i}\right)\right. \\
& \wedge\left(\bigvee _ { s ^ { \prime } \leq w < s } \chi _ { w , i _ { 0 } } ( c _ { i _ { 0 } } , x \cap c _ { i _ { 0 } } , y \cap c _ { i _ { 0 } } ) \vee \left(x \cap c_{i_{0}}=(-y) \cap c_{i_{0}}\right.\right. \\
& \left.\left.\left.\wedge \exists x \chi_{w, i_{0}}\left(c_{i_{0}}, x \cap c_{i_{0}}, y \cap c_{i_{0}}\right) \wedge \exists y \chi_{w, i_{0}}\left(c_{i_{0}}, x \cap c_{i_{0}}, y \cap c_{i_{0}}\right)\right)\right)\right) \\
& \rightarrow \operatorname{enl}(\phi(c, x, y))) .
\end{aligned}
$$

By the assumptions on $\phi(\stackrel{r}{z}, x, y)$ and on $\stackrel{r}{c}$ there is just one 1-type of $x \cap c_{i_{0}}$ over $c_{i_{0}}$ consistent with $\phi(\stackrel{r}{c}, x, y)$ such that for every $w \in W$ the formula $\exists y \chi_{w, i_{0}}\left(c_{i_{0}}, x \cap c_{i_{0}}, y \cap c_{i_{0}}\right)$ is implied by this type. The same holds for the 1-type of $y \cap c_{i_{0}}$ over $c_{i_{0}}$, which coincides with the 1-type of $x \cap c_{i_{0}}$ over $c_{i_{0}}$, and the formula $\exists x \chi_{w, i_{0}}\left(c_{i_{0}}, x \cap c_{i_{0}}, y \cap c_{i_{0}}\right)$. Since $m \cap c_{i_{0}}$ and $d \cap c_{i_{0}}$ have this 1-type, we get

$$
\begin{aligned}
\mathfrak{A} \vDash & \exists x \bigvee_{s^{\prime} \leq w<s} \chi_{w, i_{0}}\left(c_{i_{0}}, x \cap c_{i_{0}}, m \cap c_{i_{0}}\right) \\
& \wedge \exists y \bigvee_{s^{\prime} \leq w<s} \chi_{w, i_{0}}\left(c_{i_{0}}, d \cap c_{i_{0}}, y \cap c_{i_{0}}\right) .
\end{aligned}
$$

Note that $\mathfrak{A} \vDash \neg \phi(\stackrel{r}{c}, d, m)$ and $\phi$ is equivalent to $\operatorname{enl}(\phi)$. Therefore $d \cap c_{i_{0}} \neq$ $c_{i_{0}} \backslash m$ and the claim is proved.

We now give $\left(a^{\prime \prime}, b^{\prime \prime}\right)_{A}$ case by case.

Case 1: $d \cap c_{i_{0}} \neq m \cap c_{i_{0}}$. Then

$$
\mathfrak{A} \vDash \bigvee_{i=0,1,2,4,8} \phi^{i}\left(c_{i_{0}}, d \cap c_{i_{0}}, m \cap c_{i_{0}}\right) .
$$

Assume that $\mathfrak{A} \vDash \phi^{i}\left(c_{i_{0}}, d \cap c_{i_{0}}, m \cap c_{i_{0}}\right)$.

If $i=0$ or $i=2$, take an $e^{\prime}$ such that $0 \subset e^{\prime} \subset c_{i_{0}} \cap m \cap(-d)$, and $\left(a^{\prime \prime}, b^{\prime \prime}\right)_{A}=\left(d, b^{\prime} \backslash e^{\prime}\right)_{A}$. If $i=1$ or $i=8$, take $\left(a^{\prime \prime}, b^{\prime \prime}\right)_{A}=\left(a^{\prime}, d\right)_{A}$. Finally, if $i=4$, take $\left(a^{\prime \prime}, b^{\prime \prime}\right)_{A}=\left(d, b^{\prime}\right)_{A}$. 
Then, in each subcase, for any $x \in\left(a^{\prime \prime}, b^{\prime \prime}\right)_{\mathcal{P}(\omega)}$ we have

$$
\mathcal{P}(\omega) \vDash \operatorname{tp}(x, m / \stackrel{r}{c})=\operatorname{tp}(d, m / \stackrel{r}{c}) \text { and hence } \mathcal{P}(\omega) \vDash \neg \phi(\stackrel{r}{c}, x, m) .
$$

Case 2: $d \cap c_{i_{0}}=m \cap c_{i_{0}}$.

$\mathrm{Subcase} 2.1$ :

$$
\mathfrak{A} \vDash \exists x y\left(\phi^{012}\left(c_{i_{0}}, x \cap c_{i_{0}}, y \cap c_{i_{0}}\right) \wedge \neg \bigvee_{s^{\prime} \leq w<s} \chi_{w, i_{0}}\left(c_{i_{0}}, x \cap c_{i_{0}}, y \cap c_{i_{0}}\right)\right) .
$$

Since $\phi^{012}\left(c_{i_{0}}, x \cap c_{i_{0}}, y \cap c_{i_{0}}\right)$ determines the $\mathcal{L}_{\omega \omega}$-1-type of $y \cap c_{i_{0}}$ over $c_{i_{0}}$, and $m$ has the same one, we have

$$
\mathfrak{A} \vDash \exists x\left(\phi^{012}\left(c_{i_{0}}, x \cap c_{i_{0}}, m \cap c_{i_{0}}\right) \wedge \neg \bigvee_{s^{\prime} \leq w<s} \chi_{w, i_{0}}\left(c_{i_{0}}, x \cap c_{i_{0}}, m \cap c_{i_{0}}\right)\right) .
$$

There is an example $d^{\prime}$ for $x$ with $d^{\prime} \cap c_{i_{0}} \in\left(a^{\prime} \cap c_{i_{0}}, b^{\prime} \cap c_{i_{0}}\right)_{A}$, because $m \cap c_{i_{0}}=d \cap c_{i_{0}} \in\left(a^{\prime} \cap c_{i_{0}}, b^{\prime} \cap c_{i_{0}}\right)_{A}$ and hence within the given 1-type of $x \cap c_{i_{0}}$ over $c_{i_{0}}$ the formula $\phi^{i}\left(c_{i}, x \cap c_{i}, m \cap c_{i}\right)$ can be realized with some $x \cap c_{i_{0}} \in\left(a^{\prime} \cap c_{i_{0}}, b^{\prime} \cap c_{i_{0}}\right)_{A}$ for $i=0,1,2$. We can argue with $\left(d^{\prime} \cap c_{i_{0}}\right) \cup\left(d \backslash c_{i_{0}}\right)$ as with $d$ in case 1 for $i=0,1,2$.

Subcase 2.2:

$$
\mathfrak{A} \vDash \forall x y\left(\phi^{012}\left(c_{i_{0}}, x \cap c_{i_{0}}, y \cap c_{i_{0}}\right) \rightarrow \bigvee_{s^{\prime} \leq w<s} \chi_{w, i_{0}}\left(c_{i_{0}}, x \cap c_{i_{0}}, y \cap c_{i_{0}}\right)\right) .
$$

Again we have

$$
\begin{aligned}
\mathfrak{A} F \forall x y\left(\bigwedge_{s^{\prime} \leq w<s} \bigwedge_{i<r, i \neq i_{0}} \chi_{w, i}\left(c_{i}, x \cap c_{i}, y \cap c_{i}\right)\right. \\
\left.\left.\wedge \bigvee_{s^{\prime} \leq w<s} \chi_{w, i}\left(c_{i_{0}}, x \cap c_{i_{0}}, y \cap c_{i_{0}}\right)\right) \rightarrow \phi(\stackrel{r}{c}, x, y)\right) .
\end{aligned}
$$

Since

$$
\phi^{012}\left(z_{i_{0}}, x \cap z_{i_{0}}, y \cap z_{i_{0}}\right) \rightarrow \bigvee_{s^{\prime} \leq w<s} \chi_{w, i}\left(z_{i_{0}}, x \cap z_{i_{0}}, y \cap z_{i_{0}}\right),
$$

by the definition of enl we have

$$
\begin{aligned}
& \forall x y\left(\left(\bigwedge_{i<r, i \neq i_{0}} \operatorname{enl}\left(\bigwedge_{s^{\prime} \leq w<s} \chi_{w, i}\left(z_{i}, x \cap z_{i}, y \cap z_{i}\right)\right)\right.\right. \\
& \wedge\left(\bigvee _ { s ^ { \prime } \leq w < s } \chi _ { w , i } ( z _ { i _ { 0 } } , x \cap z _ { i _ { 0 } } , y \cap z _ { i _ { 0 } } ) \vee \left(x \cap z_{i_{0}}=y \cap z_{i_{0}}\right.\right. \\
& \wedge \exists x \bigvee_{s^{\prime} \leq w<s} \chi_{w, i_{0}}\left(z_{i_{0}}, x \cap z_{i_{0}}, y \cap z_{i_{0}}\right) \\
&\left.\left.\left.\left.\wedge \exists y \bigvee_{s^{\prime} \leq w<s} \chi_{w, i_{0}}\left(z_{i_{0}}, x \cap z_{i_{0}}, y \cap z_{i_{0}}\right)\right)\right)\right) \rightarrow \operatorname{enl}(\phi(\stackrel{r}{z}, x, y))\right) .
\end{aligned}
$$


In $\mathfrak{A}$ we get

$$
\begin{aligned}
\mathfrak{A} \vDash & \forall x y\left(\bigwedge_{s^{\prime} \leq w<s} \bigwedge_{i<r, i \neq i_{0}} \operatorname{enl}\left(\chi_{w, i}\left(c_{i}, x \cap c_{i}, y \cap c_{i}\right)\right)\right. \\
& \wedge\left(\bigvee _ { s ^ { \prime } \leq w < s } \chi _ { w , i } ( c _ { i _ { 0 } } , x \cap c _ { i _ { 0 } } , y \cap c _ { i _ { 0 } } ) \vee \left(x \cap c_{i_{0}}=y \cap c_{i_{0}}\right.\right. \\
& \wedge \exists x \bigvee_{s^{\prime} \leq w<s} \chi_{w, i_{0}}\left(c_{i_{0}}, x \cap c_{i_{0}}, y \cap c_{i_{0}}\right) \\
& \left.\left.\left.\left.\wedge \exists y \bigvee_{s^{\prime} \leq w<s} \chi_{w, i_{0}}\left(c_{i_{0}}, x \cap c_{i_{0}}, y \cap c_{i_{0}}\right)\right)\right)\right) \rightarrow \operatorname{enl}(\phi(\stackrel{r}{c}, x, y))\right) .
\end{aligned}
$$

As in the first subcase, we get

$$
\begin{aligned}
\mathfrak{A} \vDash & \exists x \bigvee_{s^{\prime} \leq w<s} \chi_{w, i_{0}}\left(c_{i_{0}}, x \cap c_{i_{0}}, m \cap c_{i_{0}}\right) \\
& \wedge \exists y \bigvee_{s^{\prime} \leq w<s} \chi_{w, i_{0}}\left(c_{i_{0}}, d \cap c_{i_{0}}, y \cap c_{i_{0}}\right) \wedge d \cap c_{i_{0}}=m \cap c_{i_{0}} .
\end{aligned}
$$

Putting things together yields $\mathfrak{A} \vDash \operatorname{enl}(\phi(\stackrel{r}{c}, d, m))$ and hence $\mathfrak{A} \vDash$ $\phi(\stackrel{r}{c}, d, m)$, a contradiction to the choice of $d$ and $m$.

(ii) By 3.8, $\neg \operatorname{big}(s(\phi)) \rightarrow \neg \operatorname{big}(\operatorname{enl}(s(\phi)))$, and, by 3.7, enl $(\operatorname{enl}(s(\phi))) \rightarrow$ $\operatorname{enl}(s(\phi))$ is valid. Therefore (ii) follows from (i) applied to $\operatorname{enl}(s(\phi))$.

Lemma 3.9, the construction and the monotonicity of $Q_{1}^{2}$ yield:

TheOREM 3.10. For any special $\phi$,

$\mathfrak{B} \vDash \forall \stackrel{r}{z}\left(\left(“ \stackrel{r}{z}\right.\right.$ are the atoms in the generated subalgebra" $\left.\wedge \neg \operatorname{big}(s(\phi))\left(\begin{array}{l}r \\ z\end{array}\right)\right)$

$$
\left.\rightarrow \neg Q_{1}^{2} x y s(\phi(\stackrel{r}{z}, x, y))\right) .
$$

Finally, we show how to get Theorem 3.10 for $\phi$ instead of $s(\phi)$.

Theorem 3.11. For any special $\phi$ $\mathfrak{B} \vDash \forall \underset{z}{r}\left(\left(\right.\right.$ “ $\underset{z}{r}$ are the atoms in the generated subalgebra" $\left.\wedge \neg \operatorname{big}(\phi)\left(\begin{array}{l}r \\ z\end{array}\right)\right)$

$$
\left.\rightarrow \neg Q_{1}^{2} x y \phi(\stackrel{r}{z}, x, y)\right) \text {. }
$$

Proof (by induction on $\operatorname{card}(R(\phi)))$. If $R(\phi)=\emptyset$, then $\phi(\stackrel{r}{z}, x, y) \rightarrow$ $x=y$, and hence $\mathfrak{B} \vDash \neg Q_{1}^{2} x y \phi(\stackrel{r}{c}, x, y)$.

Now assume $\mathfrak{B} \vDash \forall z$ (( “ $z$ are the atoms in the generated subalgebra" $\left.\wedge \neg \operatorname{big}(\psi)(\stackrel{r}{z})) \rightarrow \neg Q_{1}^{2} x y \psi(\stackrel{r}{z}, x, y)\right)$ for all $\psi$ with $R(\psi) \subset R(\phi)$. We show $\mathfrak{B} \vDash Q_{1}^{2} x y \phi(\stackrel{r}{c}, x, y) \rightarrow \operatorname{big}(\phi)(\stackrel{r}{c})$ for any $r$-tuple $\stackrel{r}{c}$ that consists of atoms in the generated subalgebra. Assume $\mathfrak{B} \vDash Q_{1}^{2} x y \phi(\stackrel{r}{c}, x, y)$ and let $H$ be an uncountable homogeneous set for $\phi(c, x, y)$ in $\mathfrak{B}$. By recursion on $i \leq r$ we define uncountable subsets $H^{(i)}, 0 \leq i \leq r$. 
Set $H^{(0)}:=H$. Assume $H^{(i)}$ is defined. We distinguish two cases:

Case 1: $\left\{x \cap c_{i} \mid x \in H^{(i)}\right\}$ is uncountable. Then take $H^{(i+1)} \subseteq H^{(i)}$ such that $H^{(i+1)}$ is uncountable and for any $x, y \in H^{(i+1)}$, if $x \neq y$ then $x \cap c_{i} \neq y \cap c_{i}$.

Case 2: $\left\{x \cap c_{i} \mid x \in H^{(i)}\right\}$ is countable. Then there is some $x \in H^{(i)}$ such that $\left\{y \in H^{(i)} \mid x \cap c_{i}=y \cap c_{i}\right\}$ is uncountable. Let $H^{(i+1)}$ be such a set.

For $i \notin R,\left\{x \cap c_{i} \mid x \in H^{(i)}\right\}$ is a singleton, and we are in case 2. Now consider $H^{(0)}, H^{(1)}, \ldots, H^{(r)}$. If for all $i \in R$ case 1 is true, then $H^{(r)}$ shows $\mathfrak{B} \vDash Q_{1}^{2} x y s(\phi(\stackrel{r}{c}, x, y))$. By 3.10, $\mathfrak{B} \vDash \operatorname{big}(s(\phi(\stackrel{r}{c})))$. Since $s(\phi) \rightarrow \phi$, $\mathfrak{B} \vDash \operatorname{big}(\phi(\stackrel{r}{c}))$.

If there is some $i \in R$ with case 2 being true, fix such an $i$. Then $H^{(i+1)}$ shows $\mathfrak{B} \vDash Q_{1}^{2} x y\left(\phi \wedge x \cap z_{i}=y \cap z_{i}\right)(\stackrel{r}{c}, x, y)$. Take $\psi=\phi \wedge x \cap z_{i}=y \cap z_{i}$. Then $\psi$ is also special. Since $\psi \rightarrow \phi$ and $i \in R(\phi) \backslash R(\psi)$, we have $R(\psi) \subset$ $R(\phi)$. By induction hypothesis, we conclude from $\mathfrak{B} \vDash Q_{1}^{2} x y\left(\phi \wedge x \cap z_{i}=\right.$ $\left.y \cap z_{i}\right)(\stackrel{r}{c}, x, y)$ that $\mathfrak{B} \vDash \operatorname{big}(\psi(\stackrel{r}{c}))$ and hence $\mathfrak{B} \vDash \operatorname{big}\left(\phi\left(\begin{array}{r}r \\ c\end{array}\right)\right)$.

Acknowledgement. The results are a part of the author's doctoral thesis. I would like to thank my thesis advisor H.-D. Ebbinghaus for his friendly support.

\section{References}

[Bal-Ku] J. Baldwin and D. W. Kueker, Ramsey quantifiers and the finite cover property, Pacific J. Math. 90 (1980), 11-19.

[Ba] J. E. Baumgartner, Chains and antichains in $\mathcal{P}(\omega)$, J. Symbolic Logic 45 (1980), 85-92.

[Ba-Ko] J. E. Baumgartner and P. Komjáth, Boolean algebras in which every chain and antichain is countable, Fund. Math. 111 (1981), 125-133.

[Bü] G. Bürger, The $\mathcal{L}^{<\omega}$-theory of the class of Archimedian real closed fields, Arch. Math. Logic 28 (1989), 155-166.

[Ko] P. Koepke, On the elimination of Malitz quantifiers over archimedian real closed fields, ibid., 167-171.

[Mag-Mal] M. Magidor and J. Malitz, Compact extensions of L(Q) (part 1a), Ann. Math. Logic 11 (1977), 217-261.

[Mil] H. Mildenberger, Zur Homogenitätseigenschaft in Erweiterungslogiken, Dissertation, Freiburg 1990.

[Ot] M. Otto, Ehrenfeucht-Mostowski-Konstruktionen in Erweiterungslogiken, Dissertation, Freiburg 1990.

[Ro-Tu] P. Rothmaler and P. Tuschik, A two cardinal theorem for homogeneous sets and the elimination of Malitz quantifiers, Trans. Amer. Math. Soc. 269 (1982), 273-283.

[Ru] M. Rubin, A Boolean algebra with few subalgebras, interval Boolean algebras and retractiveness, ibid. 278 (1983), 65-89. 
[Sh] S. Shelah, On uncountable Boolean algebras with no uncountable pairwise comparable or incomparable sets of elements, Notre Dame J. Formal Logic 22 (1981), 301-308.

MATHEMATISCHES INSTITUT

UNIVERSITÄT BONN

BERINGSTR. 4

D-5300 BONN 1, GERMANY

Received 8 April 1991;

in revised form 24 July 1991, 18 February 1992 and 14 September 1992 